\title{
Cytological observation on the effects of pollen irradiation in diploid and polyploid crops
}

\author{
E. M. Borrino, P. D. S. Caligari, \\ W. Powell, I. H. McNaughton \\ and A. M. Hayter.*
}

Scottish Crop Research Institute, Pentlandfield, Roslin, Midlothian EH25 9RF

\begin{abstract}
A preliminary study of the effects of pollen irradiation on the cytology of subsequent generations has been conducted on crosses within three crop groups: brassica, barley and potatoes. The hybrid chromosome complement of progeny from an interspecific cross in brassica supports previous observations (in Nicotiana and wheat) of a conventional fertilisation event following poilen irradiation. Cytological examination of M1 mitosis in brassica and potatoes revealed the frequent occurrence of aneuploidy and structural rearrangements. However, aneuploidy was not found in barley and the structural re-arrangements detected were less frequent and less extensive than reported in other species. Mitosis and meiosis of M1 and M2 generations of barley were screened for aberrations. Fertility of the M1 was reduced but was restored in the M2 of plants which were not carrying a translocation.
\end{abstract}

\section{INTRODUCTION}

In 1981, Caligari, Ingram and Jinks reported limited gene transfer in Nicotiana rustica by means of irradiated pollen. Their results and those of Pandey $(1975,1978)$ suggested that in crosses using irradiated pollen only a portion of the paternal genome was expressed in the derived progeny.

Davies (1981) enunciated the potential advantages to plant breeders of such a technique, which would enable the transfer of single or small numbers of characteristics from one parent to another within two generations. Recent work on barley, Hordeum vulgare, (Powell, Caligari and Hayter, 1983) and on wheat, Triticum aestivum, (Snape, Parker, Simpson, Ainsworth, Payne and Law, 1983) has confirmed that second generation progenies (M2) derived from crosses using irradiated pollen are more maternal in appearance than the hybrid obtained from unirradiated pollen.

Various mechanisms to explain the phenomenon of limited gene transfer have been proposed. Pandey put forward a mechanism of gene transfer which involved the incorporation of paternal DNA fragments into the egg nucleus by "pseudofertilisation" followed by a parthenogenetic doubling of the egg genome. However, a

\footnotetext{
* Present address: Sinclair, McGill (R and D) Ltd., Yonderton
} Farm, Dalrymple, Ayrshire KA6 7HT cytogenetic study of an interspecific cross in Nicotiana (Werner, Dunkin, Cornish and Jones, 1984) established that the irradiated progeny were the products of a conventional fertilisation mechanism, not parthenogenesis. In the same study the Ml progeny of an intraspecific cross of N. rustica were screened cytologically. Aneuploids and evidence of chromosomal rearrangements were frequently observed. Similar results were obtained from a cytological investigation of the effects of pollen irradiation in wheat (Snape et al., 1983).

Snape et al., proposed a "meiotic sieve" acting at or after Ml meiosis to limit transmission of paternal genes. They suggested that either, damaged paternal chromosomes are selectively lost during meisosis, or their presence results in pollen or zygote lethality. Werner et al., however, believe that most of the maternal trends observed in the M2 can be explained as the expression of mutational damage although they also state that post-meiotic selection may be involved.

The purpose of this study is to contribute towards an understanding of the mechanism(s) involved in the phenomenon of "limited gene transfer". If an effective gametophytic selection mechanism is operating then the technique of pollen irradiation has obvious applications in plant breeding. Alternatively, if the observed 
phenomenon is merely an expression of mutational damage then the technique will be of more limited value

Previous cytogenetical studies of the progeny of irradiated pollen crosses have been on Nicotiana $(2 n=4 x=48)$ and wheat, $T$. aestivum $(2 n=6 x=$ $42)$. In this study, observations are made on diploid barley, Hordeum vulgare $(2 n=2 x=14)$, on potato, Solanum tuberosum $(2 n=4 x=48)$ and on crosses at various ploidy levels within the Brassiceae.

\section{MATERIALS AND METHODS}

Cytological observations were made on three crop groups. In all three groups the corresponding unirradiated hybrid generations (F1 or F2) were sampled as control material. The parental cultivars were also screened for chromosomal abnormalities. Irradiation was by exposure to $\gamma$-rays emitted from a Cobalt 60 source.

Brassiceae (B. napus, B. campestris and Raphanus sativus)

Two types of cross were attempted following pollen irradiation. B. napus-swedes and forage rapes- $(2 n=4 x=38)$ was crossed with $B$. campestris $(2 n=2 x=20)$ and with Raphanus sativus (both $2 n=2 x=18$ and $2 n=4 x=36$ forms). Thè former cross is an easy hybridisation with normal pollen while the latter is a difficult cross, true hybrids being very rare.

In $B$. napus the swede cultivars Magres, Marian, Ruta Øtofte and Sator Øtofte were crossed with the $B$. campestris club-root differential ECD 04. The B. napus forage rape cultivar Emerald and the swede cultivars, noted above, were crossed with $R$. sativus S.C.R.I. selection RS $15(2 x)$ and cultivar Crail $(4 x)$.

Pollen was exposed to radiation doses in the range 10 to $20 \mathrm{Krads}$. B. napus cultivars were emasculated and irradiated or unirradiated pollen applied to stigmas. Subsequently samples of seed from the $20 \mathrm{Krad}$ exposure and the unirradiated control were germinated and root tips from seedlings were sampled for mitotic analysis. Roots were pretreated in $\alpha$-bromonapthalene/saponin solution for 2 hours, fixed in 3:1 alcohol: glacial acetic acid, hydrolysed in $1 \mathrm{~N} \mathrm{HCl}$ at $60^{\circ} \mathrm{C}$ for 10 minutes and stained with $1 \%$ crystal violet.

\section{Barley (Hordeum vulgare)}

The cultivar Triumph was used as female parent and Tweed as male parent. Female emasculation, pollen irradiation, pollination and embryo rescue techniques were as described previously (Powell et al., 1983). Radiation doses of 0.75 to $1.5 \mathrm{Krads}$ were used. The $\mathrm{Ml}$ generation was grown in early spring (1983), whilst the M2 was grown over winter the same year.

Roots of M1 plantlets were collected for mitotic analysis from peat blocks. Seed of the M2 and F2 generations were germinated on filter paper and primary roots sampled. Root tips were pretreated in a saturated solution of $\alpha$-bromonapthalene for 4 hours. Fixation was in glacial acetic acid; hydrolysis in $1 \mathrm{~N} \mathrm{HCl}$ at $60^{\circ} \mathrm{C}$ for 10 minutes and staining in feulgen. Counts were established from a minimum of three well spread cells in at least two different roots. Meiosis was examined in pollen mother cells (pmcs) from inflorescences fixed in 3:1 alcohol:glacial acetic acid. Hydrolysis and staining were as described for mitotic preparations.

\section{Potatoes (Solanum tuberosum)}

Three crosses were made among the commercial cultivars Pentland Ivory, Cara and Desirée. Fresh pollen was exposed to radiation doses in the range 5 to 20 Krads.

Roots were sampled from small tubers and pretreated in 8-hydroxyquinoline for 3 hours at $18^{\circ} \mathrm{C}$. Fixation was in $1 \mathrm{~N} \mathrm{HCl}$ at $60^{\circ} \mathrm{C}$. The root tip was tapped out in $45 \%$ acetic acid, a coverslip mounted and $1 \%$ crystal violet introduced.

\section{RESULTS}

\section{Brassiceae crosses}

The interspecific cross: $B$. napus $\times$

B. campestris.

The majority of Ml plants had a chromosome number the same as, or close to, that of the triploid F1 $(2 n=3 x=29)$ rather than that of the female parent $(2 n=4 x=38)$, as shown in table 1 . This indicated that the irradiated pollen derived

Table 1 Mitotic chromosome counts of the B. napus $\times$ B. campestris progeny

\begin{tabular}{lllllllllllll}
\hline Dose & $\begin{array}{l}\text { No. of } \\
\text { plants }\end{array}$ & \multicolumn{1}{c}{ Chromosome numbers } \\
\hline & & & 25 & 26 & 27 & 28 & 29 & 30 & 38 & 58 \\
Unirradiated & 58 & - & - & - & - & 56 & 1 & - & 1 \\
$20 \mathrm{Kr}$ & 52 & 2 & 2 & 1 & 23 & 20 & 1 & $3^{\prime}$ & - \\
\hline
\end{tabular}

${ }^{1}$ These plants appeared morphologically identical to $B$. napus and therefore could have been accidental selfs. 
material was hybrid in constitution and not parthenogenetic in origin. Approximately half the M1 seedlings examined were hypoploid with up to 4 chromosomes missing from the Fl complement. Structural re-arrangements such as novel telocentric chromosomes, were frequently observed. Aneuploidy in the untreated Fl was rare $(<2$ per cent $)$.

\section{The intergeneric cross: B. napus $\times$ Raphanus sativus}

Small numbers of seed were obtained from both control and irradiated material. Both diploid and tetraploid pollen crosses produced seedlings with $2 n=38$ chromosomes. These plants showed no morphological Raphanus characters and since such plants were obtained from both treated and untreated pollinations, it was assumed they were matromorphs. Indeed, matromorphic plants are known to occur in quite high numbers from $B$. napus $\times R$. sativus (untreated pollen) crosses. However, the Ml plants are being retained to see if electrophoretic techniques can reveal any evidence of gene transfer.

\section{The barley cross}

Thirteen plantlets were derived from the irradiated material; 9 from a $0.75 \mathrm{Krad}$ exposure and two each from the 1.0 and $1.5 \mathrm{Krad}$ treatments.

M1 mitosis: Mitotic counts of the parents and the hybrid control $(F 1)$ showed that these plants were euploid i.e., $2 n=2 x=14$. Reliable counts of $2 n=$ 14 were also obtained for 11 of the Ml plants. Although somatic counts were not obtained for two further plants, meiotic observations later confirmed that these plants were also euploid.

MI meiosis: Cytological screening of parents and F1 confirmed that the plants formed seven
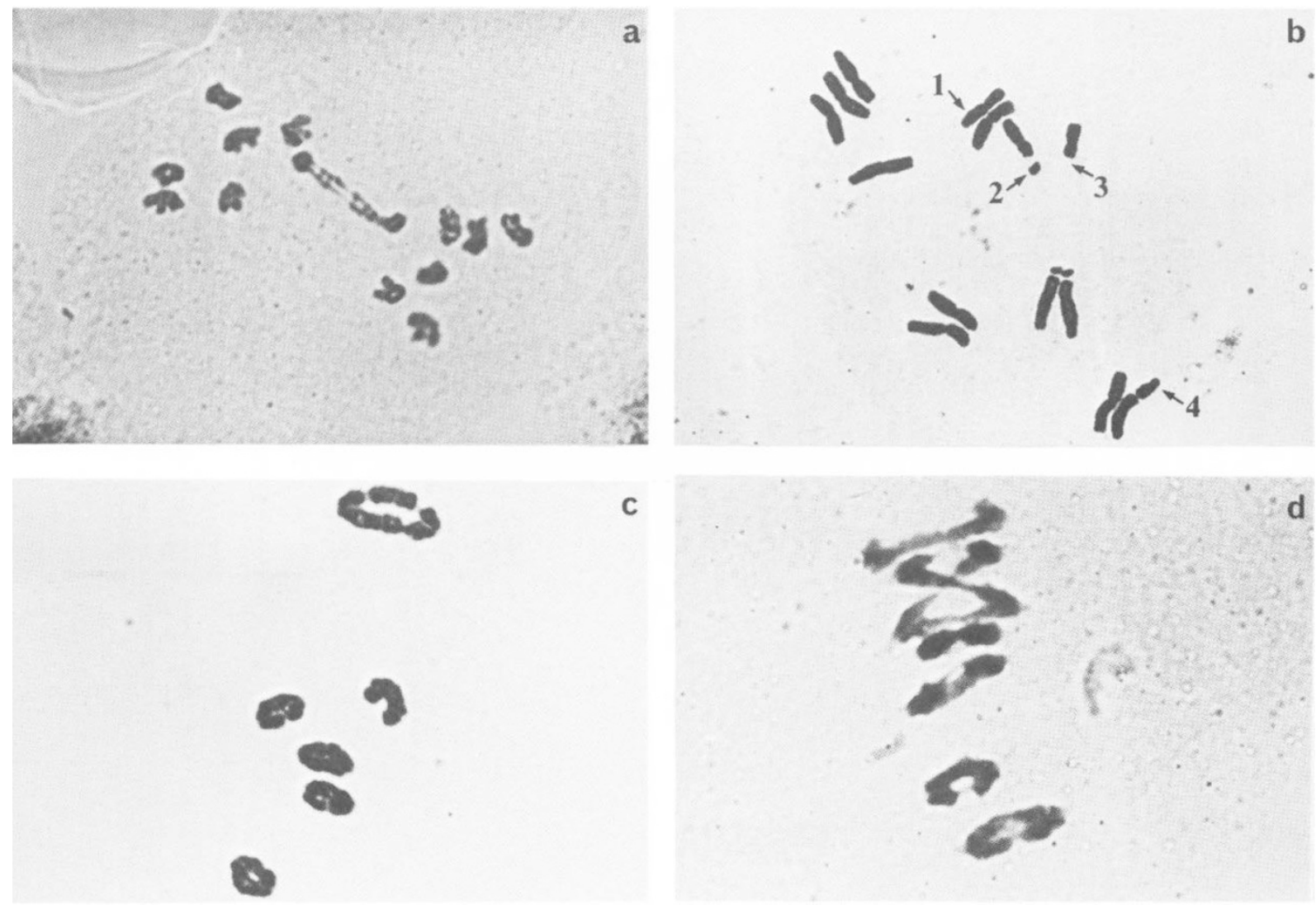

Figure 1 Mitotic and meiotic configurations in irradiated pollen derived progeny of barley. (a) bridge at AI of M1 meiosis. (b) M2 mitosis. Chromosome complement of an M1/2 derived barley plant showing structural rearrangement. Arrows 1 and 2 indicate two normal chromosomes whilst 3 and 4 show their translocated homologues. (c) and (d) M2 meiosis in a translocation heterozygote derived from plant $\mathrm{M} 1 / 2$. (c) shows an open ring quadrivalent and five bivalents. (d) shows an alternate ring quadrivalent and five bivalents. 
bivalents. In most pollen mother cells (pmcs) the bivalents formed rings held together by distal chiasmata. Some cells contained one or rarely two rod bivalents.

Twelve MI plants were examined at metaphase I of meiosis. Nine of these displayed the normal meiotic configurations observed in the Fl pmcs. The remaining three plants, all derived from a $0.75 \mathrm{Krad}$ exposure, produced multivalents. Two of the three plants showed a single quadrivalent in most pmcs, though a number of cells contained seven bivalents. The third plant gave rise to pmcs with either two quadrivalents and three bivalents, or less, frequently, a quadrivalent, a trivalent three bivalents and a univalent.

Bridges without fragments were observed at anaphase I in all Ml plants (fig. 1a). In pmcs containing bridges (varying from 20-80 per cent of pmcs among plants) usually only one and occasionally two bivalents were involved. Similar bridges were observed at a much lower frequency ( $\simeq 1$ per 2 anthers) in the FI material. However environmental factors cannot be completely eliminated as a cause of this phenomenon since the Fl material was not sown at exactly the same time as the Ml material.

Anaphase I (AI) segregations in the Ml were regular. However, a low frequency of laggards occurred (at AI and AII) in the three interchange plants and in one other plant ( $\mathrm{Ml} / 8$ ).

$M 1$ fertility: An estimate of fertility was made by comparing $\mathrm{Ml}$ and $\mathrm{Fl}$ seed set (column 1, table 2 ). The plant which had been the most cytologically aberrant (M1/9) was completely sterile. Fertility of the other two interchange plants $(\mathrm{M} 1 / 2$ and $\mathrm{Ml} / 3$ ) was greatly reduced. There were also 4 plants $(\mathrm{Ml} / 6, \mathrm{Ml} / 7, \mathrm{Ml} / 8$ and $\mathrm{Ml} / 1 \mathrm{l})$ in which no structural rearrangements were detected but which had low seed set. Only three plants had seed set similar to that of $\mathrm{F} 1$.

M2 mitosis: The karyotypes of four M2's from each M1 plant were examined. Only two cytologically abnormal plants were observed. One plant (derived from $\mathrm{Ml} / 3$ ) was trisomic whilst a second plant (from $\mathrm{Ml} / 2$ ) showed the interchange detected at meiosis of its parent (fig. Ib). There has clearly been an unequal interchange, probably between chromosome 5 (the smallest chromosome) and chromosome 6 (the shortest satellited chromosome). Both aberrant plants were from parents which showed interchange configurations at meiosis.

M2 meiosis: Meiosis was screened in a minimum of three M2's from each M1 plant. Progeny of plant $\mathrm{M} 1 / 13$ (which was not itself examined at
Table 2 Fertilities of control and irradiation derived plants as reflected by grain number per main tiller

\begin{tabular}{llll}
\hline & $\begin{array}{l}\text { Radiation } \\
\text { dose } \\
\text { (Krads) }\end{array}$ & $\begin{array}{l}\text { M1 } \\
\text { Grain number } \\
\text { (main tiller) }\end{array}$ & $\begin{array}{l}\text { M2 } \\
\text { Mean grain } \\
\text { (main tiller) }\end{array}$ \\
\hline Triumph & 0 & $\bar{x}=20$ & \\
Tweed & 0 & $\bar{x}=27$ & \\
F1/F2 & 0 & $\overline{F 1}=26$ & $\bar{F} 2=24$ \\
M1/1 & 0.75 & 26 & 27 \\
M1/2* & 0.75 & 4 & 15 \\
M1/3* & 0.75 & 8 & 18 \\
M1/4 & 0.75 & 17 & 24 \\
M1/5 & 0.75 & 18 & 23 \\
M1/6 & 0.75 & 7 & 24 \\
M1/7 & 0.75 & 12 & 26 \\
M1/8 & 0.75 & 13 & 19 \\
M1/9 & 0.75 & STERILE & -19 \\
M1/10 & 1.00 & 25 & 21 \\
M1/11 & 1.00 & 11 & 22 \\
M1/12 & 1.50 & 22 & 22 \\
M1/13* & 1.50 & 13 & 8 \\
\hline
\end{tabular}

* Plants known to be segregating at M2 for an interchange and therefore, segregating for fertility.

meiosis) were segregating for a quadrivalent. Presumably M1/13 itself was a translocation heterozygote. Six Ml/13 progeny were screened and five were interchange heterozygotes. Quadrivalents were also seen in the progeny of $\mathrm{Ml} / 2$ (figs. 1c and $\mathrm{d}$ ) and $\mathrm{Ml} / 3$. Of the three $\mathrm{Ml} / 2$ progeny studied at meiosis, two were interchange heterozygotes. This segregation was reversed in the $\mathrm{Ml} / 3$ progeny where two plants were homozygous (for either the translocated or normal chromosomes) and one was an interchange heterozygote. Clearly, these samples of M2 families are too small for significance to be attached to the segregation ratios of the interchanges. A low frequency of laggards ( $<5$ per cent) was observed at AI and AII of all M2 translocation heterozygotes. Apart from a low frequency $(<2$ per cent of pmcs) of anaphase bridges, no other cytological abnormalities were detected in the M2 progeny of the nine plants which did not carry translocations.

M2 fertility: Estimates of fertility in the M2 showed, that in the progenies with no translocations, the M2 compared well with the F2 (table 2). However in the progenies which are known to be segregating for interchanges there was a correlated segregation for fertility. Plants known to be interchange heterozygotes had fertilities $<50$ per cent of the F2. Known homozygous progeny of interchange heterozygotes had fertilities closer to the F2, but they tended to be lower than other M2 values. 


\section{Potato crosses}

Because of the vegetative nature of potatoes (i.e., the need to alternate true seed and tuber derived generations) and problems of sterility, work on this crop has progressed slowly. The parental cultivars showed the expected chromosome number $(2 n=4 x=48)$ but an aneuploid plant $(2 n=47)$ was found amongst the $19 \mathrm{~F} 1$ progeny examined. Seven aneuploids $(2 n=46$ to 49$)$ were found in 22 M1 plants for which reliable counts were obtained. Although these results are preliminary they do suggest that aneuploidy is induced by the pollen irradiation technique when used on tetraploid potato material.

\section{DISCUSSION}

The triploid rather than tetraploid karotype of $\mathrm{Ml}$ progeny from the interspecific Brassica cross $(B$. napus $\times B$. campestris) shows that the $\mathrm{M} 1$ are products of a conventional fertilisation not parthenogenic doubling as proposed by Pandey (1978). The high level of aneuploidy (50 per cent) in this cross and the structural abnormalities observed parallel the findings in the irradiated pollen derived material of Nicotiana species (Werner et al.) and wheat (Snape et al.). The preliminary screening of intervarietal Solanum tuberosum crosses also provide evidence of irradiation induced aneuploidy.

The intergeneric cross (B. napus $\times R$. sativus) generated small numbers of matromorphs in both the $\mathrm{Ml}$ and $\mathrm{Fl}$. The phenotype of the matromorphs was entirely maternal and it appears unlikely that there is any paternal contribution to the M1 progeny. However it is intended to screen these plants by electrophoretic techniques to see if any evidence of gene transfer can be detected.

The cytological effects of pollen irradiation in barley contrast markedly with those in the other species studied. Aneuploidy is absent in the M1 and rare in the M2 ( $<2$ per cent), 9 of the $13 \mathrm{Ml}$ plants investigated were euploid and showed no evidence of any structural re-arrangement. This should be compared with findings in wheat and Nicotiana where a large majority of the plants showed some cytological abnormalities.

Work on pollen irradiation in wheat (Snape et al.) showed that high levels of hypoploids and much lower levels of hyperploids were generated. It is probable that the type of damage which generates aneuploid gametes does occur in barley pollen which, like wheat, is trinucleate at the time of pollen irradiation. However the absence of aneu- ploidy in the Ml barley sample is readily explained by the lack of tolerance of hypoploidy in true diploids and the fact that the radiation induced generation of hyperploids is rare.

The bridges that were observed at AI of Ml meiosis were only rarely seen at M2 meiosis, the frequency of their occurrence at Ml was not correlated with a reduction in seed set and the absence of accompanying fragments makes it seem unlikely that they are due to the presence of inversions.

The relatively low proportion of barley plants showing evidence of structural re-arrangement at M1 is further reduced at M2; of the four M1 interchange heterozygotes, one was sterile and two of the three plants that produce M2 families had greatly reduced seed set. Without selection in the M1, only one quarter of the M2 families showed any detectable cytological abnormality and because aberrant individuals within these families had reduced fertilities, their contribution to further generations would be small and could readily be selected against. The reduced fertility of Ml individuals in which no evidence for structural change was observed (e.g., M1/6) could possibly be due to cryptic chromosomal damage which is being selected against at the gametic or zygotic level. However the resultant M2 progenies show a restoration of seed set to a normal level. Thus, this study has confirmed the findings in Nicotiana that pollen irradiation (using the technique described by Caligari et al., 1981) is followed by a true hybrid fertilisation and also that aneuploidy is frequently induced in the Ml of polyploid species. However, in diploid barley, aneuploidy was not observed, presumably because of pollen or zygote inviability. Evidence of structural re-arrangement was found in a proportion of the progeny of all three species examined.

The investigation reported here is a preliminary one and future cytological analysis will be conducted in conjunction with a programme of phenotypic assessment so that direct correlations between cytology and phenotype can be made. Also more subtle changes in the M1 and M2 chromosome complement of barley may be detected by the use of C-banding techniques and analysis of chiasma frequency.

Although barley chromosomes are more suitable for cytogenetic analysis, further screening of potato and brassica material remains necessary to elucidate the effects that differing ploidy levels are having on the outcome of pollen irradiation.

Acknowledgments We thank Dr G. H. Jones for his advice and critical comment and gratefully acknowledge the technical 
assistance of Mrs K. Liddell. We also thank Professor W. Duncan and Dr J. Law of the Western General Hospital, Edinburgh for providing the irradiation facilities.

\section{REFERENCES}

CALIGARI, P. D. S., INGRAM, N. R. AND JINKS, J. L. 1981. Gene transfer in Nicotiana rustica by means of irradiated pollen. I. Unselected progenies. Heredity, 47, 17-26.

DAVIES, D. R. 1981. Gene transfer in plants. Nature, 291, 531532.

JINKS, J. L., CALIGAR1, P. D. S. ANI) INGRAM N. R, 1981. Gene transfer in Nicotiana rustica using irradiated pollen. Nature, 291, 586-588.
PANDEY, K. K. 1975. Sexual transfers of specific genes without gametic fusion. Nature, 256, 310-31.

PANDEY, K. K. 1978. Gametic gene transfer in Nicotiana by means of irradiated pollen. Genetica, 49, 53-69.

POWELI, W., (:AI.ICIARI, P. I). S. ANI) HAYTER, A. M. 1983. The use of pollen irradiation in plant breeding. Theor. and Appl. Genet., 65, 73-76.

SNAPE, J. W., PARKER, B. B., SIMPSON, E., AINSWORTH, C. C., PAYNE, P. I. ANI) LAW, (. N. 1983. The use of irradiated pollen for differential gene transfer in wheat (Triticum aestivum). Theor. and Appl. Genet., 65, 103-111.

WERNER, C. P., DUNKIN, I. M., CORNISH, M. A. AND JONES, G. H. 1984. Gene transfer in Nicotiana rustica by means of irradiated pollen. II Cytogenetical consequences. Heredity, $52,113-119$ 